Modern Physics Letters A, Vol. 8, No. 26 (1993) 2527

(C) World Scientific Publishing Company

\title{
ERRATUM
}

\section{A CANDIDATE FOR EXACT CONTINUUM DUAL THEORY FOR SCALAR QED 3}

\author{
[Mod. Phys. Lett. A, Vol. 8, No. 14 (1993) 1343-1355]
}

A. Kovner, P. Kurzepa and B. Rosenstein

The following line should be added in the Acknowledgments:

The work of B.R. was supported by grant No. NSC-82-0208-M-001-116. 